\title{
Linhas de montagem: revisão da literatura e oportunidades para pesquisas futuras
}

\author{
Glauco Garcia Martins Pereira da Silva ${ }^{a *}$, Dalvio Ferrari Tubino ${ }^{\mathrm{b}}$, Silene Seibel $^{\mathrm{c}}$ \\ a*glauco.silva@ufsc.br, UFSC, Brasil \\ bdalvio.tubino@ufsc.br, UFSC, Brasil \\ ssilene@silene.com.br, UDESC, Brasil
}

\begin{abstract}
Resumo
Este artigo tem por objetivo identificar as principais características de pesquisa no tema linhas de montagem, a fim de propor oportunidades para trabalhos futuros. Para tanto, foram revisadas 226 publicações relevantes sobre o tema nos últimos 50 anos (1960-2010) e analisados os principais assuntos, métodos e conteúdos dos trabalhos estudados. Como resultado, observa-se uma concentração de publicações em balanceamento e sequenciamento de linhas de montagem através de métodos de modelagem e experimentos computacionais, além de significativa quantidade de estudos de linhas de modelos mistos, justificada pela crescente demanda por variedade e customização dos produtos. Existe um desafio para a pesquisa de linhas de montagem que é traduzir para os modelos gerados a complexidade dos aspectos reais do funcionamento e configurações das linhas de montagem existentes. Diversos autores enfatizam dificuldades para obter modelos aplicáveis em diversos casos e situações específicas da realidade. Os autores apontam que existem decisões estratégicas importantes para as configurações das linhas que antecedem a utilização dos modelos disponíveis. Conclui-se que há oportunidades para trabalhos científicos relacionados às pesquisas práticas e aplicadas que envolvam casos e problemas reais, além de para uma abordagem mais ampla do tema, considerando os aspectos multidisciplinares das linhas de montagem e seu relacionamento com as estratégias de produção adotadas pelas empresas.
\end{abstract}

Palavras-chave

Linha de montagem. Processos de montagem. Revisão da literatura. Bibliometria.

\section{Introdução}

Linhas de montagem são sistemas de produção orientados para o fluxo que originalmente foram desenvolvidos para a produção industrial de grandes quantidades de produtos padronizados e que vêm ganhando crescente importância para a produção de pequenos volumes de produtos customizados (Becker \& Scholl, 2006; Scholl \& Becker, 2006; Scholl et al., 2010), na medida em que o mercado exige maior variedade de produtos.

A linha de montagem pode ser definida como uma série de estações de trabalho de montagem manual ou automatizada, pelas quais um ou mais produtos são montados sequencialmente (Carnahan et al., 2001). 0 produto montado é transportado sucessivamente por cada estação através de algum dispositivo de transporte específico como, por exemplo, esteiras rolantes (Becker \& Scholl, 2006; Scholl \& Becker, 2006; Boysen et al., 2007, 2008).

De acordo com Salveson (1955) e Wild (1972), a primeira linha de montagem foi introduzida por Eli Whitney durante a revolução francesa para produção de espingardas. Esse tipo de sistema produtivo foi popularizado por Henry Ford em 1913, quando desenvolveu a primeira linha de montagem com acionamento mecânico para a produção do automóvel Ford Modelo T (Duguay et al., 1997; Amen, 2001; Klampfl et al., 2005; Boysen et al., 2008). Através dessa concepção de sistema produtivo, Ford obteve ganhos significativos em termos de produtividade e redução de custos, aspectos fundamentais para atender o modelo de consumo existente na época, baseado na comercialização de produtos de baixo preço e 
com pouca variedade (Hounshell, 1984). Nasceu aí o conceito de produção e consumo em massa, marco da segunda revolução industrial (Piore \& Sabel, 1984). A partir da linha de montagem desenvolvida por Ford, e seu indiscutível sucesso na sociedade capitalista de consumo massivo até meados do século $X X$, as linhas de montagem foram amplamente adotadas para os bens duráveis complexos, inicialmente na indústria automobilística e de produtos elétricos e posteriormente na indústria de produtos eletrônicos (Piore \& Sabel, 1984). Assim, a produção em massa ajudou a reduzir enormes custos em muitos tipos de indústria por um longo período (Jin \& Wu, 2003).

Ultimamente, os consumidores vêm desenvolvendo gostos e preferências diferenciados, aliados às constantes e rápidas evoluções em termos de conceitos, tecnologia, moda, dentre outros. lsso faz com que as empresas de determinados segmentos busquem, além de preço e qualidade, a competição baseada em flexibilidade, variedade, rapidez e confiabilidade de entrega dentro de suas estratégias (Paiva et al., 2004). De acordo com Gaither \& Frazier (1999), empresas competitivas oferecem produtos com maior valor agregado pelo menor custo e com menor tempo de resposta. Para isso, não basta que as empresas produzam com alta qualidade e baixo custo, mas também precisam ser as primeiras a levar os produtos e serviços aos clientes. Esse ambiente de mercado aliado à crescente competitividade pressiona empresas de manufatura a aperfeiçoar efetivamente seus sistemas produtivos no menor tempo possível (Zacharia \& Nearchou, 2010). Esses sistemas devem ser capazes de: i) produzir rapidamente novos modelos de produtos; ii) ajustar rapidamente as capacidades; iii) integrar tecnologia no processo; e iv) produzir grande variedade de produtos em quantidades imprevisíveis (Tasan \& Tunali, 2008). As empresas precisam expandir os limites de seus produtos para atender às expectativas dos clientes por um alto grau de customização, além de manter altos níveis de qualidade a um baixo custo (Simaria \& Vilarinho, 2004), o que torna o projeto de uma linha de montagem eficiente um aspecto de considerável importância na indústria (Baudin, 2002) e a complexidade das possíveis soluções torna o estudo do tema uma relevante oportunidade de pesquisa.

A fabricação de produtos discretos está associada à manufatura de bens em linhas de montagem. A eficácia das linhas de montagem no passado era obtida exclusivamente com base no grande volume e alta padronização, a chamada produção em massa. As mudanças no mundo dos negócios, onde a demanda é direcionada pelo consumidor, têm motivado empresas a adotar sistemas de manufatura baseados em montagem para produção sob encomenda e produção em lote, a fim de criar maior variedade de produtos (Uddin et al., 2010). Nesse contexto é possível afirmar que o ambiente externo de mercado exerce forte influência no ambiente interno das empresas, em especial nas linhas de montagem. 0 projeto de suas configurações tem características multidisciplinares, compostas de áreas de estudo de diferentes origens, que vêm sofrendo mudanças e ajustes ao longo do tempo, conforme as necessidades do mercado e os avanços tecnológicos, organizacionais e sociais. Entre essas áreas podem-se citar: recursos humanos, planejamento e controle de produção, ergonomia, automação industrial, pesquisa operacional, tecnologia da informação, qualidade, dentre outras.

Dada a importância das linhas de montagem na estratégia de produção das empresas, seus aspectos multidisciplinares e a diversidade de combinações de soluções possíveis em suas configurações, este artigo apresenta uma análise da literatura sobre o tema linhas de montagem para identificar as principais abordagens e oportunidades para pesquisa existentes e assim direcionar futuros trabalhos teóricos e práticos no assunto. Para tanto, na seção 2 são apresentados os procedimentos metodológicos adotados. Na seção 3 são descritas as principais abordagens de pesquisa disponíveis dentro do tema, com base em uma análise geral estatística dos últimos 50 anos, além de uma breve análise sobre a literatura acadêmica nacional. Já na seção 4 são apresentadas e discutidas as oportunidades de pesquisa identificadas, aprofundando-se no conteúdo dos artigos, e, por fim, na seção 5 são apresentadas as conclusões finais do artigo.

\section{Procedimentos metodológicos}

0 levantamento bibliográfico foi realizado no em novembro de 2011, com o objetivo de selecionar as principais publicações no tema linhas de montagem. Para esse fim foi utilizado um método de três etapas, desenvolvido com base no processo de pesquisa na gestão da produção e operações proposto por Miguel et al. (2010), conforme apresentado na Figura 1.

A primeira etapa do método de revisão bibliográfica diz respeito à seleção das fontes de pesquisa: foram selecionadas as bases de dados Scopus ${ }^{\mathrm{TM}}$ e Web of Science ${ }^{\circledR}$, onde estão indexados importantes periódicos da área de engenharia de produção e gestão de operações. Além das bases de dados, foram selecionadas quatro áreas de conhecimento, a saber: Ciências Sociais Aplicadas, Ciências Sociais, Engenharias e Multidisciplinar.

Devido às características multidisciplinares do tema, a varredura horizontal (segundo passo) priorizou a identificação de artigos com foco principal no tema 


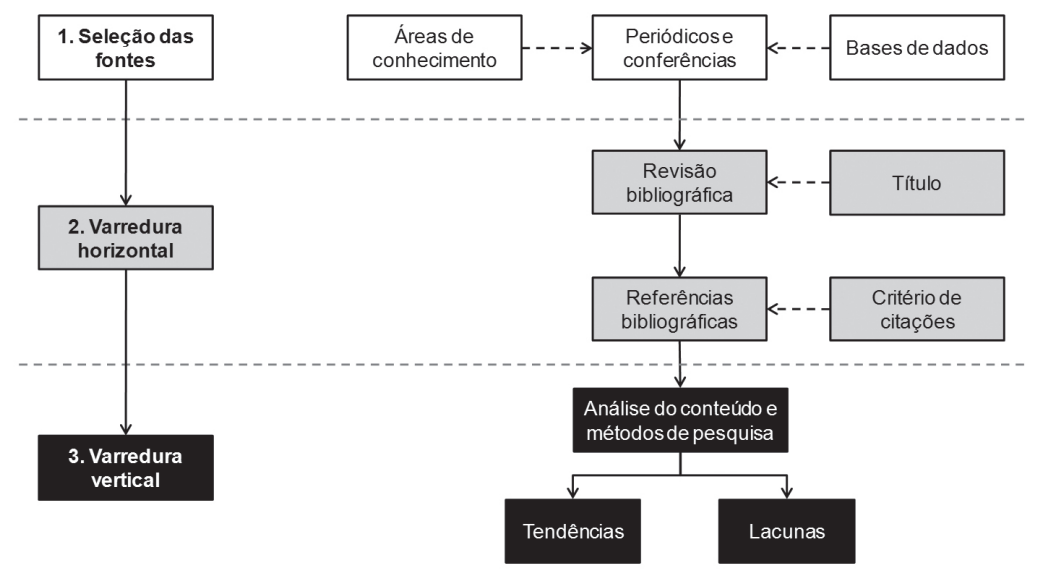

Figura 1. Método de revisão bibliográfica utilizado.

linha de montagem. Inicialmente, a pesquisa utilizou como critérios de busca os termos assembly line nos campos: título, resumo, e palavras-chave das publicações, o que resultou em numerosos artigos não pertencentes às áreas de engenharia de produção e gestão de operações. Portanto, com o intuito de selecionar apenas publicações das áreas citadas, foi realizada nova busca considerando-se apenas o campo título como filtro. Nessa primeira varredura foram encontradas 2.384 publicações na base $\operatorname{Scopus}^{\mathrm{TM}}$ e 1.570 na base Web of Science ${ }^{\circledR}$, totalizando 3.954 publicações. A partir do elevado número de artigos obtidos, a seleção das publicações foi refinada pelo critério de citações, a fim de selecionar as publicações mais utilizadas como referência para artigos até então publicados no tema linhas de montagem.

Para publicações anteriores ao ano 2000 foi estabelecido o critério de pelo menos 30 citações em outras publicações. Para o período mais recente, de 2000 a 2004, o parâmetro classificatório foi de pelo menos dez citações. Já no período de 2005 até 2010, devido ao tempo reduzido de publicação dos artigos, utilizou-se como critério classificatório o Fator de Impacto (FI) dos últimos 5 anos (5-Year Impact Factor), fator esse publicado no periódico Journal of Citations Reports (JCR) de 2011. Os periódicos que tiveram artigos publicados com os termos assembly line em seu título no período que compreende os anos de 2005 a 2010 são: Expert Systems with Applications, International Journal of Production Economics, European Journal of Operational Research, Computers and Operations Research, Computers and Industrial Engineering, Journal of Intelligent Manufacturing, Applied Mathematical Modeling, International Journal of Flexible Manufacturing Systems, International Journal of Production Research, International Journal of Advanced Manufacturing Technology e European Journal of Industrial Engineering.
Na varredura horizontal foram selecionados 226 artigos, sendo que desse total 124 (54,9\%) foram publicados no período de 2005 a 2010; 52 (23,0\%), no período de 2000 a 2004 ; e $50(22,1 \%)$ antes do ano 2000; conforme ilustra a Tabela 1.

A partir da varredura horizontal foi feita uma análise criteriosa dos artigos para identificarem-se as principais características dos estudos e futuras oportunidades de pesquisas sobre o tema. Para esse fim, inicialmente foi realizada uma análise geral através de estatísticas sobre os assuntos abordados e métodos utilizados nos artigos selecionados. Em seguida foi desenvolvida uma análise mais aprofundada do conteúdo dos artigos, com o intuito de prover uma triangulação entre essas análises e fortalecer as conclusões e oportunidades de futuras pesquisas no assunto linhas de montagem. As conclusões e oportunidades de pesquisa estão limitadas à amostra de artigos detalhada nesta seção, que reflete a literatura acadêmica mais referenciada no tema linhas de montagem.

\section{Revisão da literatura}

\subsection{Análise dos assuntos abordados}

Para retratar as principais características de pesquisa no tema linhas de montagem primeiramente foi realizada uma análise referente aos assuntos tratados dentro do tema, com o objetivo de identificar os principais aspectos abordados nas publicações. Nesse sentido foram observados título, palavras-chave, resumo e o corpo do texto das publicações, sendo que em cada artigo foram observados no máximo quatro assuntos diferentes. Por exemplo, no artigo A multidecision genetic approach for workload balancing of mixed-model U-shaped assembly line systems (Hwang \& Katayama, 2009) foram identificados 
quatro assuntos: i) Balanceamento de linha; ii) Linha de montagem de modelos mistos; iii) Layout em U; e iv) Just-in-time. Os assuntos dos respectivos artigos foram codificados e registrados em uma planilha relacionada ao banco de artigos, onde estão listadas as 226 publicações.

Um resumo dos principais tópicos abordados nas publicações pode ser encontrado na Tabela 2 e, como pode ser observado, existe uma forte concentração de publicações sobre o assunto balanceamento de linha, com 73,01\% das publicações analisadas. 0 assunto balanceamento de linha foi introduzido por Bryton (1954). Porém, a primeira publicação a respeito do tema foi feita por Salveson (1955), que introduziu o termo Problema de Balanceamento de Linha (Assembly Line Balancing Problem - ALBP) (Amen, 2001; Boysen \& Fliedner, 2008; Toksari et al., 2010). Desde então, o balanceamento de linha tem sido o principal assunto pesquisado dentro do tema linhas de montagem.

Considerando que os ALBP são classificados como NP complexos (NP Hard, Non-deterministic Polynomial-time hard) dentro dos problemas de otimização combinatória (Winch et al., 2007; Kilincci \& Bayhan, 2008; Tasan \& Tunali, 2008; Ege et al.,

Tabela 1. Resumo das referências bibliográficas selecionadas.

\begin{tabular}{cccc}
\hline Período & Critério & $\begin{array}{c}\text { Número de } \\
\text { publicações }\end{array}$ & $\begin{array}{c}\text { Porcentagem } \\
(\%)\end{array}$ \\
\hline $2010-2005$ & Fl (5 anos) >1 & 124 & 54,9 \\
$2004-2000$ & $>10$ citações & 52 & 23,0 \\
$1999-1960$ & $>$ 30 citações & 50 & 22,1 \\
\multicolumn{2}{l}{ Total de publicações } & 226 & 100,0 \\
\hline
\end{tabular}

2009; Fattahi \& Salehi, 2009; Yeh \& Kao, 2009; Zacharia \& Nearchou, 2010), muitos pesquisadores têm buscado o desenvolvimento de algoritmos eficientes, heurísticas computacionais e métodos exatos para obter soluções ótimas para os problemas de balanceamento de linha.

Alguns exemplos de técnicas empregadas nos trabalhos encontrados na revisão bibliográfica foram: algoritmos genéticos (Leu et al., 1994; Rubinovitz \& Levitin, 1995; Kim et al., 1996; Suresh et al.,1996; Hyun et al., 1998; Bautista et al., 2000; Ponnambalam et al., 2000; Rekiek et al., 2000; Sabuncuoğlu et al., 2000; Carnahan et al., 2001; Ji et al., 2001; Gonçalves \& De Almeida, 2002; Jin et al., 2002; Mansouri, 2005; Haq et al., 2006; Levitin et al., 2006; Tseng \& Tang, 2006; Wong et al., 2006; Nearchou, 2008; Venkatesh \& Dabade, 2008; Gao et al., 2009; Zhang \& Gen, 2009; Akgündüz \& Tunali, 2010); modelos de programação linear (Peeters \& Degraeve, 2006; Mahdavi et al., 2009; Moon et al., 2009; Wu \& Ji, 2009); algoritmos heurísticos (Gamberini et al., 2009; Choi et al., 2010); busca tabu (Chiang, 1998; McMullen, 1998; Pastor et al., 2002; Özcan \& Toklu, 2009; Özcan, 2010); entre outros.

Outro assunto em destaque diz respeito ao tipo de linha de montagem voltada para a produção de modelos mistos (Mixed-model), que foi abordado em 62 publicações - 27,43\% do total. As chamadas linhas de montagem de modelos mistos são linhas de produção capazes de produzir grande diversidade de produtos em pequenos lotes, com o mínimo de custo de setup entre um modelo de produto e outro

Tabela 2. Assuntos dos artigos analisados.

\begin{tabular}{|c|c|c|c|c|c|}
\hline Assunto & Artigos & $\%$ & Assunto & Artigos & $\%$ \\
\hline Balanceamento de linha & 165 & 73,01 & Sistema de apoio à decisão & 2 & 0,88 \\
\hline Modelos mistos & 62 & 27,43 & Ajuda mútua & 1 & 0,44 \\
\hline Seqüenciamento de linha & 50 & 22,12 & Buffer & 1 & 0,44 \\
\hline Just in time & 18 & 7,96 & Controle automatizado & 1 & 0,44 \\
\hline Simple Assembly Line Balancing Problem & 18 & 7,96 & Constant Work in Process & 1 & 0,44 \\
\hline Estações paralelas & 15 & 6,64 & Ergonomia & 1 & 0,44 \\
\hline Layou em formato "U” & 13 & 5,75 & Esforço físico & 1 & 0,44 \\
\hline Linha de montagem de dois lados & 13 & 5,75 & Lean & 1 & 0,44 \\
\hline Indústria automotiva & 7 & 3,10 & Linha de embalagem & 1 & 0,44 \\
\hline Pessoas & 6 & 2,65 & Linha de montagem de eletrônicos & 1 & 0,44 \\
\hline Linhas de montagem flexíveis & 5 & 2,21 & Bowl phenomenon & 1 & 0,44 \\
\hline PCB (Placas de Circuito Impresso) & 4 & 1,77 & Linhas de montagem híbridas & 1 & 0,44 \\
\hline General Assembly Line Balancing Problem & 3 & 1,33 & Linhas múltiplas & 1 & 0,44 \\
\hline Projeto de linha de montagem & 3 & 1,33 & Otimização do posto de trabalho & 1 & 0,44 \\
\hline Robotização & 3 & 1,33 & Pequenos lotes & 1 & 0,44 \\
\hline Conversão de linhas em células & 2 & 0,88 & Polivalência & 1 & 0,44 \\
\hline Linhas automatizadas & 2 & 0,88 & Sicronização de máquinas & 1 & 0,44 \\
\hline Nivelamento da produção & 2 & 0,88 & Sistema puxado & 1 & 0,44 \\
\hline Qualidade & 2 & 0,88 & Variação de demanda & 1 & 0,44 \\
\hline
\end{tabular}


(Korkmazel \& Meral, 2001). Assim, torna-se possível maior responsividade às flutuações repentinas da demanda, sem a necessidade de manter altos níveis de estoque em processo (Rahimi-Vahed \& Mirzaei, 2007; Rabbani et al., 2008). Esse tipo de configuração de linha permite produzir uma variedade de produtos para atender às necessidades do mercado, característica presente no cenário atual do consumo (Uddin et al., 2010).

Com o objetivo de aumentar as vendas e os lucros, as empresas estão sob constante pressão para aumentar o número de variações ou modelos em um produto básico. A linha de montagem de modelos mistos permite a produção de modelos variados com características similares, envolvendo diferentes tarefas, métodos e tempos padrão, o que requer diferentes instalações, componentes e matérias-primas. Esse tipo de solução de montagem vem crescendo na indústria a fim de atender a tendência atual de diversificação na demanda do consumidor (Ponnambalam et al., 2003; Simaria \& Vilarinho, 2004).

Segundo Rahimi-Vahed \& Mirzaei (2007), existem dois principais problemas associados à implantação de linhas de montagem de modelos mistos. 0 primeiro diz respeito ao projeto e balanceamento da linha e o segundo, à determinação da sequência de produção para os diferentes modelos. Por esse motivo, o assunto sequenciamento de produção foi bastante explorado com 50 publicações, contabilizando 22,12\% dos artigos. Nos artigos de sequenciamento também foram propostos modelos matemáticos, heurísticos e de simulação para determinar o sequenciamento que proporcionasse melhor eficiência na utilização das linhas (Erel et al., 2007; Guo et al., 2008; Javadi et al., 2008; Sabuncuoğlu et al., 2008; Fattahi \& Salehi, 2009; Akgündüz \& Tunali, 2010; Choi et al., 2010; Emde et al., 2010; Özcan, 2010; Özcan et al., 2010; Uddin et al., 2010).

\subsection{Análise dos procedimentos de pesquisa utilizados}

A segunda característica analisada nos artigos revisados diz respeito ao procedimento metodológico de pesquisa utilizado, segundo as tipologias propostas por Filippini (1997): i) Estudo de campo; ii) Estudo de caso; iii) Experimento; iv) Modelagem; v) Simulação; vi) Survey; vii) Teórico-conceitual. É importante destacar que os artigos que adotaram mais de um procedimento metodológico foram enquadrados nos respectivos tipos. Como, por exemplo, o artigo Absalom: Balancing assembly lines with assignment restrictions (Scholl et al., 2010), que faz uso de modelagem para tratar o problema de balanceamento de linha e de experimentos computacionais para testar a solução proposta. Nesse caso, o artigo foi enquadrado nos procedimentos do tipo Modelagem e Experimento. 0 resultado assim obtido apresenta forte concentração de certos tipos de procedimento de pesquisa para abordar o tema linhas de montagem, como ilustra a Tabela 3.

0 estudo demonstrou que o maior foco da abordagem metodológica está na utilização de Modelagem (85,0\%) e Experimento (77,9\%). Essa concentração é explicável: conforme demonstrado no item 3.1, os pesquisadores utilizam esses procedimentos para pesquisas na solução dos problemas de balanceamento e sequenciamento de linha, para os quais esses procedimentos metodológicos mais se aplicam. Existe uma forte tendência de tratar problemas práticos por meio do desenvolvimento de modelos matemáticos e heurísticos aliados à realização de experimentos computacionais, com o objetivo de testar a eficácia desses modelos.

Em contrapartida, foi encontrado um número reduzido de publicações que adotaram metodologias com base empírica para o estudo do tema linhas de montagem, como Estudos de caso, surveys (empíricas), e Estudos de campo. Os estudos de caso encontrados, em sua maioria, foram assim classificados por utilizarem dados de casos para modelagem matemática, porém sem discussões com base em aplicações práticas e intervenções nos sistemas reais de produção.

\subsection{Pesquisa sobre linhas de montagem no Brasil}

Os artigos e análises até então apresentados têm embasamento na literatura acadêmica internacional sobre linhas de montagem, o que reflete a realidade da pesquisa no tema fora do país. 0 Brasil, ainda considerado um país voltado para a manufatura - com diversas montadoras automobilísticas e de variados setores instalando suas plantas no país - também carece de pesquisa e desenvolvimento no assunto, para que possa melhorar a eficiência em suas linhas atuais e futuras. Por esse motivo, nesse item é apresentada uma breve revisão da literatura nacional até então publicada.

Utilizou-se o mesmo procedimento metodológico de revisão da literatura apresentado na Figura 1,

Tabela 3. Resumo da abordagem metodológica dos artigos analisados.

\begin{tabular}{lcc}
\hline Procedimento de pesquisa & Artigos & Porcentagem (\%) \\
\hline Modelagem & 192 & 85,0 \\
Experimento & 176 & 77,9 \\
Simulação & 30 & 13,3 \\
Estudo de caso & 25 & 11,1 \\
Teórico-conceitual & 13 & 5,7 \\
Survey & 7 & 3,1 \\
Estudo de campo & 2 & 0,9 \\
\hline
\end{tabular}


com algumas simplificações necessárias. Na etapa de seleção das fontes de pesquisa foi considerada a base de dados SciElo Brasil (Scientific Eletronic Library Online), onde estão indexados os principais periódicos científicos brasileiros. Em novembro de 2011 procedeu-se à etapa de varredura horizontal para seleção das publicações a analisar. A primeira busca considerou os termos linha de montagem no campo título, porém não foram encontradas publicações. Por esse motivo foi necessário ampliarem-se os critérios de busca, a fim de obterem-se resultados significativos, utilizando somente o termo montagem e o campo assunto como filtro, obtendo-se como resultado 21 publicações. Após análise do conteúdo dos artigos no contexto das áreas de engenharia de produção e gestão de operações, foram selecionadas sete publicações. Sendo que com o número reduzido de artigos não foi necessário refinar a seleção pelo critério de citações, tomando-se para análise todas as sete publicações.

Definidas as publicações de referência, procedeu-se à etapa de varredura vertical através da análise do conteúdo dos artigos e dos procedimentos de pesquisa utilizados, segundo a classificação proposta por Filippini (1997). 0 Tabela 4 traz um resumo destas análises.

Apesar do número inferior de artigos nacionais analisados, quando comparados à literatura internacional em linhas de montagem, identificou-se um quadro semelhante. Das sete publicações selecionadas, cinco tratam do tema balanceamento de linha, reforçando a concentração de publicações no tema. 0 procedimento de pesquisa mais utilizado foi o de Modelagem - em quatro aplicações, seguido de Experimento e Estudo de caso - com três aplicações, e de Simulação - com duas ocorrências. Tais resultados são semelhantes aos observados nos itens 3.1 e 3.2, onde se observou a carência de estudos práticos e aplicados a problemas reais, sendo que os estudos de caso observados não discutem as soluções de organização e configurações das linhas em si, mas têm como objetivo a discussão dos modelos desenvolvidos.
Os trabalhos analisados fazem parte das principais publicações sobre o tema linhas de montagem no Brasil, que carece de pesquisas e desenvolvimento no assunto. 0 aumento do número de empresas que migram para o país visando operar atividades de montagem e localizar suas instalações próximas ao mercado consumidor brasileiro reforça a relevância dos estudos no tema. Dessa maneira, as análises e sugestões para a pesquisa sobre linhas de montagem são estendidas para trabalhos acadêmicos e pesquisas no país.

\section{Oportunidades para pesquisas futuras}

A partir da análise das características de pesquisa no tema linhas de montagem foi identificada forte concentração de estudos focados no balanceamento e sequenciamento de linhas através dos métodos Modelagem e de experimentos computacionais. Contudo, existe um grande desafio para esse tipo de pesquisa que é o de traduzir para os modelos desenvolvidos os aspectos reais do funcionamento e das configurações das linhas de montagem existentes, a fim de se ter o melhor resultado possível.

Diversos autores enfatizam tanto a dificuldade de se obterem bons modelos, que possam ser aplicados, quanto a carência de estudos práticos. No trabalho de Özcan (2010), em que se propõe um algoritmo para o balanceamento de linhas de montagem de dois lados, o autor afirma que nos modelos existentes os tempos das tarefas são assumidos como determinísticos. Entretanto, salienta que na vida real, especialmente em linhas de montagem manuais, os tempos de execução das tarefas podem variar, como em uma distribuição de probabilidades. Essa variação dos tempos pode resultar de quebra de máquinas, pouca motivação das pessoas, falta de treinamento e qualificação dos operadores, complexidade das tarefas, fatores ambientais, dentre outros; aspectos difíceis de serem modelados matematicamente.

Os autores Zhang \& Gen (2009) apontam a dificuldade de estabelecer o tempo de ciclo baseado na demanda de diferentes modelos de produtos. Eles afirmam que as diferenças de experiência e habilidade

Tabela 4. Análise da literatura brasileira pesquisada.

\begin{tabular}{|c|c|c|}
\hline Artigo & Assunto(s) & Procedimento(s) de pesquisa \\
\hline Guimarães et al. (1993) & $\begin{array}{l}\text { Sequenciamento de operações e } \\
\text { automatização }\end{array}$ & Modelagem e Experimento \\
\hline Santoro \& Moraes (2000) & Balanceamento de linha & Simulação e Estudo de caso \\
\hline Fernandes \& Dalalio (2000) & $\begin{array}{l}\text { Balanceamento de linha, grupos } \\
\text { autogerenciados e demanda variável }\end{array}$ & Modelagem e Experimento \\
\hline Naveiro \& Guimarães (2003) & Análise biomecânica & Estudo de caso \\
\hline Souza et al. (2003) & $\begin{array}{l}\text { Balanceamento de linha e linhas } \\
\text { multimodelos }\end{array}$ & Simulação e Estudo de caso \\
\hline Fernandes et al. (2008) & Balanceamento de linha & Modelagem e Estudo de caso \\
\hline Silva Junior et al. (2010) & Balanceamento de linha & Modelagem e Experimento \\
\hline
\end{tabular}


dos operadores, o tempo de processamento de uma tarefa e os custos operacionais - como salários - variam entre os operadores. E, portanto, a definição do operador mais adequado para cada tarefa, para reduzir o tempo de ciclo e assim aumentar a eficiência da linha e reduzir os custos totais, torna-se um problema extremamente complexo de ser modelado. Por outro lado, Jolai et al. (2009) apontam o balanceamento de linha como um problema multidisciplinar e afirmam que métodos algorítmicos e heurísticos não são efetivos para solucioná-los, propondo uma abordagem baseada na metodologia Análise de Envoltória de Dados (DEA).

Os autores Lucertini e Paccearelli (1998) demonstraram que, apesar da existência de centenas de trabalhos publicados sobre projetos de linhas de montagem, apenas um pequeno número de empresas utilizam as técnicas publicadas para balancear suas linhas. Os autores Rekiek et al. (2002) vão além e afirmam que os algoritmos acadêmicos são poucos utilizados nas empresas industriais devido ao fato de serem pouco efetivos e práticos, além de utilizarem poucos dados e perderem informações substanciais, resolvendo problemas fictícios ao invés de problemas industriais.

Boysen \& Fliedner (2008) realizaram em seu artigo uma ampla pesquisa na literatura existente sobre balanceamento de linha, apontando os possiveis modelos a serem utilizados para problemas práticos, com o objetivo de reduzir a lacuna entre a pesquisa e o mundo real. Os autores consideram surpreendente a lacuna existente entre a discussão acadêmica e as aplicações práticas até os dias de hoje. Eles apontam os trabalhos de Chase (1974) e Schöniger \& Spingler (1989), baseados em surveys empíricas que revelaram um número muito pequeno de empresas utilizando algoritmos matemáticos para o planejamento de configuração das linhas de montagem. Os autores afirmam que essa lacuna ainda existe, ou até aumentou. Como conclusão, constatam que em mais de 50 anos de estudo do tema, apenas 15 de 312 artigos publicados trataram explicitamente de casos reais de balanceamento de linha, o que representa menos de 5\% das publicações, e apontam esse fato como um dos indicadores da grande lacuna entre a pesquisa e os problemas reais. Os autores propõem o artigo citado como primeiro passo para reduzir essa lacuna de pesquisa no futuro e apontam como passos futuros a realização de estudos empíricos sobre problemas práticos de balanceamento de linha, para confirmar se as soluções apontadas no artigo são válidas em situações reais. Além disso, sugerem tanto que as pesquisas foquem cada vez mais a resolução de problemas reais, quanto que os periódicos publiquem mais estudos de caso sobre o assunto.

A lacuna de pesquisa identificada pelos autores é reforçada através das análises das referências bibliográficas selecionadas, que apontam a deficiência em termos de estudos empíricos e práticos sobre o assunto. Conforme apresentado na Tabela 3, entre as 226 referências foram encontradas apenas duas publicações classificadas como Estudo de campo (Simaria et al., 2009; Wu \& Ji, 2009). Entre as 25 publicações classificadas como Estudo de caso, apenas em três (Bartholdi, 1993; Álvarez et al., 2009; Cortés et al., 2010) foram realizadas interferências reais no processo, além da utilização dos dados para a modelagem matemática. Bartholdi (1993) afirma que seu trabalho foi o primeiro a apresentar dados reais em mais de 30 anos de pesquisa sobre linhas de montagem.

Outro ponto a ser destacado nas publicações analisadas é a ausência de produção acadêmica explorando soluções para o relacionamento entre os aspectos estratégicos das empresas e seus modelos de gestão da produção para as linhas de montagem. Foram encontradas poucas publicações referentes aos modelos de gestão da produção aplicados à linha de montagem, como a manufatura enxuta ou a customização em massa.

0 tema manufatura enxuta foi explorado apenas em uma publicação (Álvarez et al., 2009) e relata a aplicação da ferramenta Mapeamento de Fluxo de Valor (MFV), sistema kanban e abastecimento via milk-run. 0 conceito just-in-time foi abordado em 18 publicações (Sumichrast \& Russell, 1990; Kubiak \& Sethi, 1991; Xiaobo \& Ohno, 1997; McMullen, 1998; Zeramdini et al., 2000; Drexl \& Kimms, 2001; Kurashige et al., 2002; Jin \& Wu, 2003; Mansouri, 2005; Hwang et al., Gen, 2008; Javadi et al., 2008; Rabbani et al., 2008; Alpay, 2009; Boysen et al., 2009a, b; Hwang \& Katayama, 2009, 2010; Koulouriotis et al., 2010) que utilizaram modelos matemáticos e heurísticos para testar o nivelamento da produção, layout em U e fazer o balanceamento e sequenciamento de linhas.

Diversos autores mencionam a importância das linhas de montagem para o cumprimento das estratégias competitivas das empresas sem, no entanto, aprofundarem o tema para apontar soluções. Rekiek et al. (2002) afirmam que a montagem é a principal atividade estratégica das empresas e que questões como utilizar e desenvolver sistemas de montagem mais eficientes são fundamentais para o sucesso das empresas de manufatura. Segundo os autores, os sistemas de montagem determinam a qualidade final dos produtos e também afetam o tempo de resposta ao mercado (time-to-market), nivel de entrega, dentre outros aspectos.

Boysen et al., (2007) e Boysen \& Fliedner (2008) colocam que a individualização dos produtos é a maneira de atender às necessidades dos consumidores atuais e, portanto, os sistemas de fluxo em linha devem estar aptos a produzir em pequeno volume, em um sistema de montagem sob pedido (assembly-to-order). 
Os autores afirmam que essas características viabilizam modernas estratégias, como a customização em massa, o que torna o planejamento e implantação de sistemas de montagem de grande relevância prática no futuro.

Os autores Simaria et al., (2009) apontam que o dinamismo e a intensa competição do mercado atual, juntamente com as mudanças de tecnologia, têm levado à redução do ciclo de vida dos produtos, aumento da demanda por produtos customizados e à pressão pela diversificação do mix e por produtos com mais modelos e oferta de características opcionais. Além disso, a responsividade em termos de rapidez e confiança dos tempos de entrega é demandada pelo mercado, onde o tempo está sendo visto como um aspecto diferencial chave. Por isso, os autores afirmam que é evidente a necessidade de sistemas flexíveis capazes de produzir a variedade sem aumento significante dos custos, levando empresas a utilizarem diferentes configurações das linhas de montagem. As linhas de montagem devem atingir alta produtividade, qualidade uniforme e custos baixos, além do que devem ser flexíveis, para gerar produtos com pequeno ciclo de vida e volume, demandas variáveis e grande variedade de versões opções.

Por fim, os autores colocam que as linhas de montagem são peças importantes para a cadeia de suprimentos, suportando "estratégias" de postponement, além de terem a estrutura ideal para realizar tarefas de customização no produto final, dentro do conceito de customização em massa. Com a utilização de trabalho intensivo, elas podem estar localizadas próximas ao mercado do consumidor final. Essas constatações, dentre outras encontradas na literatura especializada no tema linhas de montagem, reforçam o papel que esse tipo de sistema produtivo tem para o cumprimento das estratégias de negócio das organizações, marcando a influência externa (mercado) nas decisões relativas às linhas de montagem. Apesar disso, poucos trabalhos exploram esse relacionamento de uma forma aprofundada, apontando como as linhas de montagem contribuem para o atendimento das estratégias competitivas estabelecidas e como devem estar estruturadas para tal. Assim, o balanceamento e sequenciamento das linhas são assuntos importantes para a obtenção de uma melhor eficiência nesse tipo de sistema produtivo. No entanto, existem decisões estratégicas na concepção, projeto e implantação das linhas que antecedem a utilização desses métodos.

No contexto estratégico, os autores Hayes et al. (1988) sugerem dez áreas de decisão dentro da estratégia de produção, que representam escolhas fundamentais para as prioridades competitivas escolhidas pelas empresas serem alcançadas, a saber: tecnologia; instalações; capacidade; integração vertical; planejamento, programação e controle de produção; organização; recursos humanos; novos produtos; qualidade; e medidas de desempenho. Essas áreas de decisão influenciam diretamente nas soluções e configurações das linhas de montagem, em seus múltiplos aspectos. Por esse motivo, Silva (2013) sugere no Tabela 5 algumas oportunidades a serem consideradas em pesquisas futuras sobre o tema, a fim de ajustar as linhas às estratégias competitivas das empresas. A maior parte das questões apresentadas leva em consideração temas pouco explorados nas publicações revisadas para o presente artigo.

Para finalizar as análises e discussões, o Tabela 6 mostra um resumo das contribuições desenvolvidas

Tabela 5. Estratégia de produção e configurações de linhas de montagem.

\begin{tabular}{|c|c|}
\hline Áreas de decisão & Aspectos a serem pesquisados \\
\hline Tecnologia & $\begin{array}{l}\text { Que tipos de recursos utilizar (pessoas, máquinas, robôs)? Qual o grau de automatização necessário? Como } \\
\text { identificar (rastrear) componentes e produtos? Como controlar o ritmo da linha? Como transportar os } \\
\text { produtos na linha? }\end{array}$ \\
\hline Instalações & $\begin{array}{l}\text { Onde instalar as linhas de montagem? Onde estão fornecedores/clientes? Utilizar centros de distribuição? } \\
\text { Quantos tipos de modelos produzir na linha? Quantas linhas adotar? Quanto investir? Qual o retorno } \\
\text { sobre os investimentos? }\end{array}$ \\
\hline Capacidade & Qual capacidade de produção a linha deve ter? Utilizar a capacidade máxima? \\
\hline Integração vertical & $\begin{array}{l}\text { Quais itens a empresa deve produzir e quais deve adquirir de terceiros? Como deve ser o relacionamento } \\
\text { com os fornecedores? }\end{array}$ \\
\hline $\begin{array}{l}\text { Planejamento, programação e } \\
\text { controle de produção }\end{array}$ & $\begin{array}{l}\text { Qual deve ser a política de atendimento (make to stock, assembly to order, make to order, engineering } \\
\text { to order)? Como fazer o balanceamento e sequenciamento da linha? Como dimensionar e onde } \\
\text { alocar buffers? Qual deve ser o tamanho dos lotes? Qual o tempo de ciclo? Onde será o ponto de } \\
\text { desacoplamento? }\end{array}$ \\
\hline Organização & $\begin{array}{l}\text { Que tipo de layout adotar? Como fazer o abastecimento de componentes, quem é o responsável, com que } \\
\text { frequência, como dimensionar? Como estabelecer o padrão de trabalho? Como estimar os tempos das } \\
\text { operações? }\end{array}$ \\
\hline Recursos humanos & $\begin{array}{l}\text { Adotar operadores multifuncionais? Qual deve ser o nível de qualificação dos operadores? Quais fatores } \\
\text { ergonômicos e de segurança devem ser considerados? Como motivar e capacitar os operadores? }\end{array}$ \\
\hline Novos produtos & $\begin{array}{l}\text { Qual o grau de customização dos produtos? Existe modularidade de peças e componentes? Existe } \\
\text { engenharia simultânea? Qual a complexidade dos produtos? Como será o ciclo de vida dos produtos? }\end{array}$ \\
\hline Qualidade & $\begin{array}{l}\text { Existem mecanismos de detecção de problemas/defeitos? Como será o monitoramento da qualidade? } \\
\text { Existem grupos de melhoria? }\end{array}$ \\
\hline
\end{tabular}


Tabela 6. Tendências e oportunidades de pesquisa.

\begin{tabular}{lll}
\hline & \multicolumn{1}{c}{ Características da pesquisa } & \multicolumn{1}{c}{ Oportunidades para trabalhos futuros } \\
\hline Assuntos chave & - Balanceamento de linha & - Problemas e situações reais \\
& - Linhas de montagem de modelos mistos & - Abordagem multidisciplinar \\
- Sequenciamento da produção & - Estratégia de produção versus configurações das linhas \\
Procedimentos de & - Modelagem (algoritmos, métodos heurísticos & - Estudo de caso \\
pesquisa & e exatos) & - Estudo de campo \\
& Experimentos computacionais & - Pesquisa ação \\
& & - Surveys empíricas \\
\hline
\end{tabular}

ao longo deste trabalho. Essas levam em consideração as características da pesquisa e as oportunidades propostas para trabalhos futuros, no que se refere aos assuntos e procedimentos de pesquisa no tema linhas de montagem.

\section{Conclusões}

As linhas de montagem foram originalmente concebidas para a produção em fluxo de grandes quantidades de produtos com pouca variedade e baixo custo, dentro do conceito de produção e consumo em massa. Nos últimos cem anos, desde sua popularização com Henry Ford, houve grandes mudanças no cenário competitivo em que as empresas operam para responder às necessidades dos consumidores. Por esse motivo, as empresas necessitam direcionar suas estratégias de produção e as configurações de suas linhas de montagem para competir com rapidez, flexibilidade, qualidade, baixo custo e com produtos variados e customizados.

Nesse sentido, este artigo procurou identificar, através da revisão de 226 artigos publicados nos últimos 50 anos, as principais características e oportunidades para pesquisas no tema, com o objetivo de instigar trabalhos futuros que auxiliem teóricos e práticos a melhor adequar as linhas de montagem e suas configurações às necessidades de mercado do ambiente competitivo no qual as empresas hoje se inserem. As discussões e conclusões apresentadas estão limitadas à análise da amostra citada, que representa a literatura mais referenciada em termos de pesquisa acadêmica em linhas de montagem. Como resultado dessa análise é possível constatar a concentração de pesquisas voltadas para o balanceamento e sequenciamento das linhas de montagem através de Modelagem e experimentos computacionais. Outro assunto em destaque é a linha de modelos mistos, o que aponta para a tendência de utilização das linhas para a produção de pequenos lotes de itens variados e customizados. Essa constatação reflete a característica atual de mercado, voltada para a diversificação, o que exige das linhas de montagem maior flexibilidade em termos de configuração e pode ser considerado um ponto importante a ser explorado em pesquisas no tema.
A partir desse cenário, o presente artigo propõe como oportunidades futuras de pesquisa o relacionamento das estratégias das empresas com as configurações das linhas de montagem através da exploração de problemas e casos reais e da utilização de procedimentos de pesquisa como Estudos de caso, Estudos de campo, Pesquisa ação e Surveys empíricas. Além de uma abordagem que envolva os aspectos multidisciplinares das configurações das linhas de montagem (p. ex., áreas de decisão: tecnologia, instalações, capacidade, integração vertical, PCP, organização, recursos humanos, produtos, qualidade, medidas de desempenho), visando prover pesquisas mais completas sobre o tema e estreitando a distância existente entre o mundo teórico e o real.

\section{Referências}

Akgündüz, 0. S., \& Tunali, S. (2010). An adaptive genetic algorithm approach for the mixed-model assembly line sequencing problem. International Journal of Production Research, 48(17), 5157-5179. http://dx.doi. org/10.1080/00207540903117857

Alpay, S. (2009). GRASP with path relinking for a multiple objective sequencing problem for a mixed-model assembly line. International Journal of Production Research, 47(21), 6001-6017. http://dx.doi. org/10.1080/00207540802158291

Álvarez, R., Calvo, R., Peña, M. M., \& Domingo, R. (2009). Redesigning an assembly line through lean manufacturing tools. International Journal of Advanced Manufacturing Technology, 43(9-10), 949-958. http:// dx.doi.org/10.1007/s00170-008-1772-2

Amen, M. (2001). Heuristic methods for cost-oriented assembly line balancing: A comparison on solution quality and computing time. International Journal of Production Economics, 69(3), 255-264. http://dx.doi. org/10.1016/S0925-5273(99)00096-1

Bartholdi, J. J. (1993). Balancing two-sided assembly lines: A case study. International Journal of Production Research, 31(10), 2447-2461. http://dx.doi. org/10.1080/00207549308956868

Baudin, M. (2002). Lean assembly: The nuts and bolds of making assembly operations flow. New york: Productivity Press.

Bautista, J., Suarez, R., Mateo, M., \& Companys, R. (2000). Local search heuristics for the assembly line balancing problem with incompatibilities between tasks, San Francisco, CA, USA. In IEEE International Conference on Robotics and Automation. 
Becker, C., \& Scholl, A. (2006). A survey on problems and methods in generalized assembly line balancing. European Journal of Operational Research, 168(3), 694715. http://dx.doi.org/10.1016/j.ejor.2004.07.023

Boysen, N., \& Fliedner, M. (2008). A versatile algorithm for assembly line balancing. European Journal of Operational Research, 184, 39-56. http://dx.doi.org/10.1016/j. ejor.2006.11.006

Boysen, N., Fliedner, M., \& Scholl, A. (2007). A classification of assembly line balancing problems. European Journal of Operational Research, 183(2), 674-693. http://dx.doi. org/10.1016/j.ejor.2006.10.010

Boysen, N., Fliedner, M., \& Scholl, A. (2008). Assembly line balancing: Which model to use when? International Journal of Production Economics, 111(2), 509-528. http://dx.doi.org/10.1016/j.jpe.2007.02.026

Boysen, N., Fliedner, M., \& Scholl, A. (2009a). Level scheduling of mixed-model assembly lines under storage constraints. International Journal of Production Research, 47(10), 2669-2684. http://dx.doi. org/10.1080/00207540701725067

Boysen, N., Fliedner, M., \& Scholl, A. (2009b). The product rate variation problem and its relevance in real world mixed-model assembly lines. European Journal of Operational Research, 197(2), 818-824. http://dx.doi. org/10.1016/j.ejor.2008.06.038

Bryton, B. (1954). Balancing of a continuos production line (Master's thesis). Northwestern University, Evanston.

Carnahan, B. J., Norman, B. A., \& Redfern, M. S. (2001). Incorporating physical demand criteria into assembly line balancing. IEEE Transactions, 33(10), 875-887. http:// dx.doi.org/10.1080/07408170108936880

Chase, R. B. (1974). Survey of paced assembly lines. Industrial Engineering, 6, 14-18.

Chiang, W. C. (1998). The application of a tabu search metaheuristic to the assembly line balancing problem. Annals of Operations Research, 77, 209-227. http:// dx.doi.org/10.1023/A:1018925411397

Choi, Y. C., Kim, Y. D., \& Bang, J. Y. (2010). Scheduling algorithms for an air conditioner manufacturing system composed of multiple parallel assembly lines. International Journal of Advanced Manufacturing Technology, 1-17.

Cortés, P., Onieva, L., \& Guadix, J. (2010). Optimising and simulating the assembly line balancing problem in a motorcycle manufacturing company: A case study. International Journal of Production Research, 48(12), 3637-3656. http://dx.doi. $\operatorname{org} / 10.1080 / 00207540902926522$

Drexl, A., \& Kimms, A. (2001). Sequencing JIT mixed-model assembly lines under station-load and part-usage constraints. Management Science, 47(3), 480-491. http://dx.doi.org/10.1287/mnsc.47.3.480.9777

Duguay, C. R., Landry, S., \& Pasin, F. (1997). From mass production to flexible/agile production. International Journal of Operations \& Production Management, 17(11-12), 11831195. http://dx.doi.org/10.1108/01443579710182936

Ege, Y., Azizoglu, M., \& Ozdemirel, N. E. (2009). Assembly line balancing with station paralleling. Computers \& Industrial Engineering, 57(4), 1218-1225. http://dx.doi. $\operatorname{org} / 10.1016 / j$.cie.2009.05.014

Emde, S., Boysen, N., \& Scholl, A. (2010). Balancing mixedmodel assembly lines: A computational evaluation of objectives to smoothen workload. International Journal of Production Research, 48(11), 3173-3191. http:// dx.doi.org/10.1080/00207540902810577

Erel, E., Gocgun, Y., \& Sabuncuoğlu, 1. (2007). Mixed-model assembly line sequencing using beam search. International Journal of Production Research, 45(22), 5265-5284. http://dx.doi.org/10.1080/00207540600806497

Fattahi, P., \& Salehi, M. (2009). Sequencing the mixed-model assembly line to minimize the total utility and idle costs with variable launching interval. International Journal of Advanced Manufacturing Technology, 45(9-10), 987998. http://dx.doi.org/10.1007/s00170-009-2020-0

Fernandes, F. C. F., \& Dalalio, A. G. (2000). Balanceamento e rebalanceamento de linhas de montagem operadas por grupos de trabalho autogerenciados. Gestão \& Produção, 73), 378-398. http://dx.doi.org/10.1590/ S0104-530X2000000300013

Fernandes, F. C. F., Godinho Filho, M., Cutigi, R. A., \& Guiguet, A. M. (2008). 0 uso da programação inteira 0-1 para o balanceamento de linhas de montagem: modelagem, estudos de caso e avaliação. Produção, 18(2), 210-221. http://dx.doi.org/10.1590/S0103-65132008000200002

Filippini, R. (1997). Operations management research: Some reflections on evolution, models and empirical studies in OM. International Journal of Operations \& Production Management, 1777-8), 655-670. http:// dx.doi.org/10.1108/01443579710175583

Gaither, N., \& Frazier, G. (1999). Production and Operations Management (8th ed). Cincinnati: South-Western College Publishing.

Gamberini, R., Gebennini, E., Grassi, A., \& Regattieri, A. (2009). A multiple single-pass heuristic algorithm solving the stochastic assembly line rebalancing problem. International Journal of Production Research, 47(8), 2141-2164. http:// dx.doi.org/10.1080/00207540802176046

Gao, J., Sun, L., Wang, L., \& Gen, M. (2009). An efficient approach for type 11 robotic assembly line balancing problems. Computers \& Industrial Engineering, 56(3), 1065-1080. http://dx.doi. org/10.1016/j.cie.2008.09.027

Gonçalves, J. F., \& De Almeida, J. R. (2002). hybrid genetic algorithm for assembly line balancing. Journal of Heuristics, 8(6), 629-642. http://dx.doi. org/10.1023/A:1020377910258

Guimarães, G., Boaventura Netto, P. 0., \& Naveiro, R. M. (1993). Geração de seqüências viáveis para montagem automatizada. Produção, 3(1), 33-44. http://dx.doi. org/10.1590/S0103-65131993000100004

Guo, Z. X., Wong, W. K., Leung, S. Y. S., Fan, J. T., \& Chan, S. F. (2008). A genetic-algorithm-based optimization model for scheduling flexible assembly lines. International Journal of Advanced Manufacturing Technology, 36(12), 156-168. http://dx.doi.org/10.1007/s00170-0060818-6

Haq, A. N., Rengarajan, K., \& Jayaprakash, J. (2006). A hybrid genetic algorithm approach to mixed-model assembly line balancing. International Journal of Advanced Manufacturing Technology, 28(3-4), 337-341. http:// dx.doi.org/10.1007/s00170-004-2373-3

Hayes, R. H., Wheelwright, S. C., \& Clark, K. B. (1988). Dynamic Manufacturing. New York: Free Press. 
Hounshell, D. A. (1984). From the american system to mass production 1800-1932. Baltimore: The Johns Hopkins University Press.

Hwang, R., \& Katayama, H. (2009). A multi-decision genetic approach for workload balancing of mixed-model U-shaped assembly line systems. International Journal of Production Research, 47(14), 3797-3822. http://dx.doi. org/10.1080/00207540701851772

Hwang, R., \& Katayama, H. (2010). Uniform workload assignments for assembly line by GA-based amelioration approach. International Journal of Production Research, 48(7), 1857-1871. http://dx.doi. org/10.1080/00207540802577953

Hwang, R., Katayama, H., \& Gen, M. (2008). U-shaped assembly line balancing problem with genetic algorithm. International Journal of Production Research, 46(16), 4637-4649. http://dx.doi. org/10.1080/00207540701247906

Hyun, C. J., Kim, Y., \& Kim, Y. K. (1998). A genetic algorithm for multiple objective sequencing problems in mixed model assembly lines. Computers and Operations Research, 25(7-8), 675-690. http://dx.doi.org/10.1016/ S0305-0548(98)00026-4

Javadi, B., Rahimi-Vahed, A., Rabbani, M., \& Dangchi, M. (2008). Solving a multi-objective mixed-model assembly line sequencing problem by a fuzzy goal programming approach. International Journal of Advanced Manufacturing Technology, 39(9-10), 975-982. http:// dx.doi.org/10.1007/s00170-007-1267-6

Ji, P., Sze, M. T., \& Lee, W. B. (2001). Genetic algorithm of determining cycle time for printed circuit board assembly lines. European Journal of Operational Research, 128(1), 175-184. http://dx.doi.org/10.1016/ S0377-2217(99)00348-3

Jin, M., \& Wu, S. D. (2003). A new heuristic method for mixed model assembly line balancing problem. Computers and Industrial Engineering, 44(1), 159-169. http://dx.doi. org/10.1016/S0360-8352(02)00190-0

Jin, Z. H., Ohno, K., Ito, T., \& Elmaghraby, S. E. (2002). Scheduling hybrid flowshops in printed circuit board assembly lines. Production and Operations Management, 11(2), 216-230. http://dx.doi. org/10.1111/j.1937-5956.2002.tb00492.x

Jolai, F., Jahangoshai Rezaee, M., \& Vazifeh, A. (2009). Multicriteria decision making for assembly line balancing. Journal of Intelligent Manufacturing, 20(1), 113-121. http://dx.doi.org/10.1007/s10845-008-0107-3

Kilincci, O., \& Bayhan, G. M. (2008). A P-invariantbased algorithm for simple assembly line balancing problem of type-1. International Journal of Advanced Manufacturing Technology, 37(3-4), 400-409. http:// dx.doi.org/10.1007/s00170-007-0975-2

Kim, Y. K., Kim, Y. J., \& Kim, Y. (1996). Genetic algorithms for assembly line balancing with various objectives. Computers and Industrial Engineering, $30(3$ SPEC. 1SS.), 397-409.

Klampfl, E., Gusikhin, O., \& Rossi, G. (2005). Optimization of workcell layouts in a mixed-model assembly line environment. International Journal of Flexible Manufacturing Systems, 17(4), 277-299.

Korkmazel, T., \& Meral, S. (2001). Bicriteria sequencing methods for the mixed-model assembly line in justin-time production systems. European Journal of
Operational Research, 131(1), 188-207. http://dx.doi. org/10.1016/S0377-2217(00)00061-8

Koulouriotis, D. E., Xanthopoulos, A. S., \& Tourassis, V. D. (2010). Simulation optimisation of pull control policies for serial manufacturing lines and assembly manufacturing systems using genetic algorithms. International Journal of Production Research, 48(10), 2887-2912. http:// dx.doi.org/10.1080/00207540802603759

Kubiak, W., \& Sethi, S. (1991). Note on "level schedules for mixed-model assembly lines in just-in-time production systems'. Management Science, 371), 121-122. http:// dx.doi.org/10.1287/mnsc.37.1.121

Kurashige, K., Yanagawa, Y., Miyazaki, S., \& Kameyama, Y. (2002). Time-based goal chasing method for mixedmodel assembly line problem with multiple work stations. Production Planning \& Control, 13(8), 735-745. http:// dx.doi.org/10.1080/0953728031000057325

Leu, Y. Y., Matheson, L. A., \& Rees, L. P. (1994). AssemblyLine balancing using genetic algorithms with heuristicgenerated initial populations and multiple evaluation criteria. Decision Sciences, 25(4), 581-606. http://dx.doi. org/10.1111/j.1540-5915.1994.tb01861.x

Levitin, G., Rubinovitz, J., \& Shnits, B. (2006). A genetic algorithm for robotic assembly line balancing. European Journal of Operational Research, 168(3), 811-825. http:// dx.doi.org/10.1016/j.ejor.2004.07.030

Lucertini, M., \& Paccearelli, D. A. P. (1998). Modeling an assembly line for configuration and flow management. Computer Integrated Manufacturing Systems, 11, 15-24. http://dx.doi.org/10.1016/S0951-5240(98)00005-6

Mahdavi, I., Javadi, B., Sahebjamnia, N., \& Mahdavi-Amiri, N. (2009). A two-phase linear programming methodology for fuzzy multi-objective mixed-model assembly line problem. International Journal of Advanced Manufacturing Technology, 44(9-10), 1010-1023. http://dx.doi.org/10.1007/s00170-008-1914-6

Mansouri, S. A. (2005). A Multi-Objective Genetic Algorithm for mixed-model sequencing on JIT assembly lines. European Journal of Operational Research, 167(3), 696716. http://dx.doi.org/10.1016/j.ejor.2004.07.016

McMullen, P. R. (1998). JIT sequencing for mixed-model assembly lines with setups using Tabu Search. Production Planning and Control, 9(5), 504-510. http://dx.doi. org/10.1080/095372898233984

Miguel, P. A. C., Fleury, A.; Mello, C. H. P.; Nakano, D. N.; Turrioni, J. B.; Ho, L. L.; Martins, R. A.; Pureza, V. M. M.; \& Morabito, R. (2010). Metodologia de pesquisa em engenharia de produção e gestão de operações. Rio de Janeiro: Elsevier.

Moon, l., Logendran, R., \& Lee, J. (2009). Integrated assembly line balancing with resource restrictions. International Journal of Production Research, 47(19), 5525-5541. http://dx.doi.org/10.1080/00207540802089876

Naveiro, R. M., \& Guimarães, C. P. (2003). Uma aplicação da Análise Biomêcânica no Processo de Montagem de Produtos Industriais. Revista Produção, 13(1).

Nearchou, A. C. (2008). Multi-objective balancing of assembly lines by population heuristics. International Journal of Production Research, 46(8), 2275-2297. http://dx.doi. org/10.1080/00207540600988089

Özcan, U. (2010). Balancing stochastic two-sided assembly lines: A chance-constrained, piecewise-linear, mixed integer program and a simulated annealing algorithm. 
European Journal of Operational Research, 205(1), 8197. http://dx.doi.org/10.1016/j.ejor.2009.11.033

Özcan, U., Cercioglu, H., Gokcen, H., \& Toklu, B. (2010). Balancing and sequencing of parallel mixed-model assembly lines. International Journal of Production Research, 48(17), 5089-5113. http://dx.doi. org/10.1080/00207540903055735

Özcan, U., \& Toklu, B. (2009). Multiple-criteria decisionmaking in two-sided assembly line balancing: A goal programming and a fuzzy goal programming models. Computers \& Operations Research, 36(6), 1955-1965. http://dx.doi.org/10.1016/j.cor.2008.06.009

Paiva, E., Carvalho, L., \& Fensterseifer, J. (2004). Estratégia de produção e de operações. Porto Alegre: Bookman.

Pastor, R., Andres, C., Duran, A., \& Perez, M. (2002). Tabu search algorithms for an industrial multi-product and multi-objective assembly line balancing problem, with reduction of the task dispersion. Journal of the Operational Research Society, 53(12), 1317-1323. http:// dx.doi.org/10.1057/palgrave.jors.2601457

Peeters, M., \& Degraeve, Z. (2006). An linear programming based lower bound for the simple assembly line balancing problem. European Journal of Operational Research, 168(3), 716-731. http://dx.doi.org/10.1016/j. ejor.2004.07.024

Piore, M. J., \& Sabel, C. F. (1984). The second industrial divide: Possibilities for prosperity. New York: Basic Books. PMid:17837177.

Ponnambalam, S. G., Aravindan, P., \& Naidu, G. M. (2000). A multi-objective genetic algorithm for solving assembly line balancing problem. International Journal of Advanced Manufacturing Technology, 16(5), 341-352. http://dx.doi.org/10.1007/s001700050166

Ponnambalam, S. G., Aravindan, P., \& Subba Rao, M. (2003). Genetic algorithms for sequencing problems in mixed model assembly lines. Computers and Industrial Engineering, 45(4), 669-690. http://dx.doi. org/10.1016/j.cie.2003.09.001

Rabbani, M., Rahimi-Vahed, A., \& Torabi, S. A. (2008). Real options approach for a mixed-model assembly line sequencing problem. International Journal of Advanced Manufacturing Technology, 37(11-12), 1209-1219. http://dx.doi.org/10.1007/s00170-007-1058-0

Rahimi-Vahed, A., \& Mirzaei, A. H. (2007). A hybrid multiobjective shuffled frog-leaping algorithm for a mixedmodel assembly line sequencing problem. Computers \& Industrial Engineering, 53, 642-666. http://dx.doi. org/10.1016/j.cie.2007.06.007

Rekiek, B., De Lit, P., \& Delchambre, A. (2000). Designing mixed-product assembly lines. IEEE Transactions on Robotics and Automation, 16(3), 268-280. http://dx.doi. org/10.1109/70.850645

Rekiek, B., De Lit, P., \& Delchambre, A. (2002). Hybrid assembly line design and user's preferences. International Journal of Production Research, 4O(5), 1095-1111. http:// dx.doi.org/10.1080/00207540110116264

Rubinovitz, J., \& Levitin, G. (1995). Genetic algorithm for assembly line balancing. International Journal of Production Economics, 41(1-3), 343-354. http://dx.doi. org/10.1016/0925-5273(95)00059-3

Sabuncuoğlu, 1., Erel, E., \& Tanyer, M. (2000). Assembly line balancing using genetic algorithms. Journal of
Intelligent Manufacturing, 11(3), 295-310. http://dx.doi. org/10.1023/A:1008923410076

Sabuncuoğlu, 1., Gocgun, Y., \& Erel, E. (2008). Backtracking and exchange of information: Methods to enhance a beam search algorithm for assembly line scheduling. European Journal of Operational Research, 186(3), 915930. http://dx.doi.org/10.1016/j.ejor.2007.02.024

Salveson, M. E. (1955). The assembly line balancing problem. Journal of Industrial Engineering, 6, 18-25.

Santoro, M. C., \& Moraes, L. H. (2000). Simulação de uma linha de montagem de motores. Gestão \& Produção, 73), 338-351. http://dx.doi.org/10.1590/S0104-530X2000000300010

Scholl, A., \& Becker, C. (2006). State-of-the-art exact and heuristic solution procedures for simple assembly line balancing. European Journal of Operational Research, 168(3), 666693. http://dx.doi.org/10.1016/j.ejor.2004.07.022

Scholl, A., Fliedner, M., \& Boysen, N. (2010). Absalom: Balancing assembly lines with assignment restrictions. European Journal of Operational Research, 200(3), 688701. http://dx.doi.org/10.1016/j.ejor.2009.01.049

Schöniger, J., \& Spingler, J. (1989). Planung der montageanlage. Technica, 14, 27-32.

Silva, G. G. M. P. (2013). Linhas de montagem e estratégias competitivas: estudo de múltiplos casos. Florianópolis: Universidade Federal de Santa Catarina (Tese de doutorado). Universidade Federal de Santa Catarina, Florianópolis.

Silva Junior, 0. C., Miranda Júnior, G., \& Conceição, S. V. (2010). Reconfiguração dinâmica de linhas de montagem. Pesquisa Operacional, 30(1), 237-258. http://dx.doi. org/10.1590/S0101-74382010000100012

Simaria, A. S., \& Vilarinho, P. M. (2004). A genetic algorithm based approach to the mixed-model assembly line balancing problem of type 11. Computers \& Industrial Engineering, 474), 391-407. http://dx.doi.org/10.1016/j. cie.2004.09.001

Simaria, A. S., Zanella De Sá, M., \& Vilarinho, P. M. (2009). Meeting demand variation using flexible U-shaped assembly lines. International Journal of Production Research, 47(14), 3937-3955. http://dx.doi. org/10.1080/00207540701871044

Souza, M. C. F., Yamada, M. C., Porto, A. J. V., \& Gonçalves Filho, E. V. (2003). Análise da alocação de mão-de-obra em linhas de multimodelos de produtos com demanda variável através do uso da simulação: um estudo de caso. Produção, 13(3), 63-77. http://dx.doi.org/10.1590/ S0103-65132003000300006

Sumichrast, R. T., \& Russell, R. S. (1990). Evaluating mixed-model assembly line sequencing heuristics for just-in-time production systems. Journal of Operations Management, 9(3), 371-390. http://dx.doi. org/10.1016/0272-6963(90)90161-6

Suresh, G., Vinod, V. V., \& Sahu, S. (1996). A genetic algorithm for assembly line balancing. Production Planning and Control, 7(1), 38-46. http://dx.doi. org/10.1080/09537289608930323

Tasan, S. 0., \& Tunali, S. (2008). A review of the current applications of genetic algorithms in assembly line balancing. Journal of Intelligent Manufacturing, 19(1), 4969. http://dx.doi.org/10.1007/s10845-007-0045-5

Toksari, M. D., Isleyen, S. K., Güner, E., \& Baykoç, Ö. F. (2010). Assembly line balancing problem with deterioration tasks and learning effect. Expert Systems 
with Applications, 37(2), 1223-1228. http://dx.doi. org/10.1016/j.eswa.2009.06.005

Tseng, H. E., \& Tang, C. E. (2006). A sequential consideration for assembly sequence planning and assembly line balancing using the connector concept. International Journal of Production Research, 44(1), 97-116. http:// dx.doi.org/10.1080/00207540500250606

Uddin, M. K., Soto, M. C., \& Lastra, J. L. M. (2010). An integrated approach to mixed-model assembly line balancing and sequencing. Assembly Automation, 30(2), 164-172. http://dx.doi.org/10.1108/01445151011029808

Venkatesh, J. V. L., \& Dabade, B. M. (2008). Evaluation of performance measures for representing operational objectives of a mixed model assembly line balancing problem. International Journal of Production Research, 46(22), 63676388. http://dx.doi.org/10.1080/00207540701383164

Wild, R. (1972). Mass-production management - The design and operation of production flow-line systems. London: Wiley.

Winch, J. K., Cai, X., \& Vairaktarakis, G. L. (2007). Cyclic job scheduling in paced assembly lines with cross-trained workers. International Journal of Production Research, 45(4), 803828. http://dx.doi.org/10.1080/00207540600635144

Wong, W. K., Mok, P. Y., \& Leung, S. Y. S. (2006). Developing a genetic optimisation approach to balance an apparel assembly line. International Journal of Advanced Manufacturing Technology, 28(3-4), 387-394. http:// dx.doi.org/10.1007/s00170-004-2350-x

$\mathrm{Wu}$, Y., \& Ji, P. (2009). A scheduling problem for PCB assembly: A case with multiple lines. International Journal of Advanced Manufacturing Technology, 43(1112), 1189-1201. http://dx.doi.org/10.1007/s00170-0081797-6
Xiaobo, Z., \& Ohno, K. (1997). Algorithms for sequencing mixed models on an assembly line in a JIT production system. Computers and Industrial Engineering, 32(1), 4756. http://dx.doi.org/10.1016/S0360-8352(96)00193-3

Yeh, D. H., \& Kao, H. H. (2009). A new bidirectional heuristic for the assembly line balancing problem. Computers \& Industrial Engineering, 57(4), 1155-1160. http://dx.doi. org/10.1016/j.cie.2009.05.004

Zacharia, P. T., \& Nearchou, A. C. (2010). Multi-objective fuzzy assembly line balancing using genetic algorithms. Journal of Intelligent Manufacturing, 1-13.

Zeramdini, W., Aigbedo, H., \& Monden, Y. (2000). Bicriteria sequencing for just-in-time mixed-model assembly lines. International Journal of Production Research, 38(15), 34513470. http://dx.doi.org/10.1080/002075400422743

Zhang, W., \& Gen, M. (2009). An efficient multiobjective genetic algorithm for mixed-model assembly line balancing problem considering demand ratio-based cycle time. Journal of Intelligent Manufacturing, 1-12.

\section{Agradecimentos}

0 primeiro autor deste artigo agradece ao Conselho Nacional de Desenvolvimento Científico e Tecnológico (CNPq) pela bolsa de pesquisa durante o curso de doutorado e à Coordenação de Aperfeiçoamento de Pessoal de Nível Superior (CAPES) pela bolsa de estágio de doutorado no exterior através do Programa de Doutorado Sanduíche no Exterior (PDSE).

\section{Assembly lines: existing work and avenues for future research Abstract}

The goal of this paper was to identify the main characteristics of assembly line research to propose avenues for future work. We reviewed 226 relevant publications written on the topic in the last 50 years (1960 to 2010) and analyzed the main issues, methods and content of these works. As a result, we found a large number of publications concerned with the balancing and sequencing of assembly lines through modeling and computational experimental methods. We also identified a trend in mixed models line studies, justified by the growing demand for product variety and customization. However, a major challenge in this type of research involves representing the complexity and practical aspects of existing assembly line operation and settings in the developed models. Several authors have emphasized the difficulty of obtaining models that can be applied to several cases and specific real situations. The authors noted that major strategic decisions for assembly line configurations have preceded the use of available models and should be studied further. We conclude by identifying avenues for scientific research on practical and applied cases involving real problems, in addition to a broader approach on the subject that could consider the multidisciplinary aspects of assembly lines and their relationship with the production strategies adopted by companies.

\section{Keywords}

Assembly line. Assembly processes. Literature review. Bibliometrics. 